\title{
Reflexiones sobre las modificaciones en la estructura y funcionamiento del Comité Editorial
}

En el año 2003 al cumplir la revista 18 años de publicación ininterrumpida, planteábamos en su editorial la disyuntiva si podíamos aspirar a ingresar a índices bibliográficos más exigentes de corriente principal (ISI-Thomas: Index of Scientific Information y MEDLINE: Index Medicus on Line) o deberíamos conformarnos con mantener y consolidar la indización ya alcanzada: SciELO (Scientific Electronic Library on Line) en 2002 y LILACS (Literatura Latinoamericana y del Caribe en Ciencias de la Salud) desde sus inicios ${ }^{I}$. Aunque es difícil para una revista biomédica nacional ingresar a ISI o a MEDLINE, este paso no es totalmente infranqueable. En efecto, en el Primer Seminario de Editores de Revistas SciELO-Chile, realizado en enero de 2006, se nos comunicó que SciELO está registrando 1,5 millones de consultas mensuales y que de las 55 revistas nacionales indizadas en SciELO, 12 ya están indizadas en ISI, y de ellas 4 lo han hecho estando previamente en SciELO. Estas cifras permiten deducir que SciELO sería la plataforma adecuada para preparar estratégicamente el paso o "salto" cualitativo siguiente que es alcanzar ISI. Para ello deberemos aumentar la cantidad y especialmente la calidad de los trabajos originales y eventualmente de series clínicas publicadas en nuestra revista. También necesitamos lograr entre nuestros socios una "masa crítica" de autores que publiquen los resultados de sus estudios no sólo en esta revista, sino también en revistas de "corriente principal" y con un factor de impacto significativo (idealmente > 2). Esto se debe a que ISI realiza un análisis de citaciones de la revista como también de los autores y del comité editorial de cada revista que postula a ser indizada alli².

Los últimos directorios de la Sociedad han dado pasos importantes en este sentido ${ }^{3,4}$, a través de la instauración de premios a los mejores artículos publicados y especialmente con la creación de concursos de proyectos de investigación (a contar de 2002) ${ }^{3}$ y más recientemente con el llamado a concurso de proyectos multicéntricos $(2005)^{4}$, que permitirán crear proyectos "semilla", que podrían eventualmente concursar con algún grado de posibilidades en las ligas mayores de los concursos de proyectos nacionales como FONIS o FONDECYT o de agencias internacionales. Los proyectos multicéntricos constituyen una oportunidad para concertar esfuerzos, ayudan a crear una masa crítica de investigadores y, por su estructuración, generan un círculo virtuoso de exposición, aprendizaje y control de procedimientos y análisis de resultados entre pares, creándose redes de investigadores afines y eventualmente multidisciplinarios, ubicados en diferentes áreas geográficas de nuestro país o de otras naciones.

Esta posibilidad que ahora tienen los miembros de la Sociedad, de realizar investigación apoyada por la misma institución, se ha constituido en una oportunidad para mejorar la calidad de la revista, ya que una de las condiciones del concurso es que los proyectos aprobados por la comisión científica deban generar una publicación en la sección "trabajos originales" de la revista. Con especial beneplácito hemos podido verificar que esta condición se está cumpliendo. En efecto, los resultados de los 6 proyectos financiados entre 2002 y 2005, han sido presentados en los congresos anuales de la Sociedad y los tres primeros han generado publicaciones en extenso en la Revista, sirviendo dos de ellos de referencia al Consenso Nacional 2005 sobre manejo de la neumonía del adulto adquirida en la Comunidad .

Por otra parte, desde comienzos de 2004 los miembros del cuerpo ejecutivo de esta revista han estado preocupados, junto con la directiva de la Sociedad Chilena de Enfermedades Respiratorias, de renovar, vigorizar y modificar el funcionamiento de la revista, a fin de superar sus debilidades y mantener sus actuales fortalezas. En la cuenta anual 2005, el directorio presidido por el Dr. Manuel Barros M., anunció la decisión de reestructurar el Comité Editorial ${ }^{4}$, a fin de agilizar el trabajo editorial y promover entre los pares la publicación en la revista, creando paulatinamente nuevas secciones y asignando tareas específicas y en plazos acotados a quienes constituyan el nuevo Comité Editorial. Se establecía en dicha cuenta que más que conseguir que un determinado especialista se hiciera cargo de una sección de la revista, estas secciones se deberían constituir en la más plena expresión del quehacer de las diversas comisiones, secciones y ramas que integran nuestra 
Sociedad. En otras palabras, el logro de metas más altas en la publicación de la revista -aumento de la calidad y cantidad de artículos originales, actualizaciones y casos clínicos, ingreso a ISI y MEDLINE, aumento de los números regulares por cada volumen-deberá sustentarse en el trabajo de cada uno de los órganos de la Sociedad. La calidad de la revista debe ser un fiel reflejo de la actividad científica desarrollada por los miembros de la Sociedad.

Existe la sensación generalizada que la revista podría alcanzar mayores niveles de calidad, en la medida que los miembros de la Sociedad se comprometan a realizar su valioso y necesario aporte. No es suficiente que lean y comenten sus artículos, sino que también deberían hacernos llegar sus propias sugerencias e inquietudes para superar las debilidades que detecten y, lo más importante, enviar artículos para su eventual publicación, que cumplan estrictamente los requisitos solicitados a los autores por la revista.

La actual directiva de la Sociedad presidida por el Dr. Raúl Corrales V., ha expresado el anhelo de aumentar la producción de la revista de 4 a 5 ó 6 números anuales y lograr indizarla en MEDLINE en el futuro cercano ${ }^{7}$. En ese contexto se ha procedido a ejecutar las modificaciones en la estructura y funcionamiento del Comité Editorial. Estas modificaciones fueron gestadas y discutidas en un proceso de análisis y reflexión en que fueron ponderadas diversas iniciativas y proposiciones planteadas en reuniones sostenidas por las dos últimas mesas directivas de la Sociedad con los editores responsables de la revista.

En base al reglamento de funcionamiento de la revista del 21 de Agosto de 1991, se elaboró una descripción de funciones del Comité Editorial y además se propuso una serie de innovaciones, entre las que destacan las siguientes:

a) Estimular el envío de artículos originales, dando algunas facilidades de secretaría y de análisis estadístico a quienes presenten buenas comunicaciones libres a nuestro congreso anual.

b) Estimular el envío de casos clínicos. A los editores de la revista nos ha llamado la atención que, siendo el trabajo clínico mayoritario y prioritario entre los miembros de la Sociedad, el número de Casos y de Series o Experiencias Clínicas enviadas para su eventual publicación es realmente muy exiguo en relación a las potencialidades de las instituciones nacionales dedicadas a las enfermedades respiratorias. Una explicación a ello podría ser que un número importante de nuestros socios carece de una adecuada orientación acerca de cuándo, cómo y por qué publicar un caso o una serie clínica. Para paliar esta situación se ha publicado en este número de la revista un artículo esclarecedor sobre este tema. . Además, se han designado "corresponsales de casos clínicos" en diversos hospitales y centros que cubran la mayor parte del país y en que se ejerce nuestra especialidad, abarcando niños, adultos y cirugía torácica. Los corresponsales de casos clínicos de la revista tendrán como función reclutar manuscritos con esta temática, revisar que cumplan con los requerimientos de la revista y enviarlos con una evaluación previa al Comité Editorial, lo cual acelerará el proceso de revisión por pares.

c) Publicar en la "Sección Actualizaciones" las actividades de la Sociedad, como conferencias, simposios y controversias, que sean consideradas por los socios como sobresalientes o de muy buen nivel. Con este sentido se ha elaborado un instructivo que facilita al autor transformar su exposición en un artículo de revisión.

d) Incorporar paulatinamente nuevas secciones. Se han propuesto las siguientes: Casos fisiopatológicos, Medicina basada en evidencias, Educación médica, Ética médica, Medicina intensiva y Cirugía de tórax.

e) Integrar a un pediatra, en la medida de lo posible, a cada una de las secciones de la revista, a fin de aumentar los artículos del ámbito pediátrico, que actualmente constituyen cerca del $30 \%$ de los artículos publicados.

Las modificaciones en la estructura y funcionamiento de la revista se iniciaron en el primer número de 2006, con la incorporación del Dr. Fernando Saldías Peñafiel como editor adjunto, quien acompañará al Dr. Rodrigo Moreno B. en esa importante y delicada función editorial. En este segundo número de 2006 se puede apreciar la constitución del nuevo Comité Editorial de la revista que se mantendrá en funciones durante el bienio 2006-2007. Los nombres de los corresponsales de Series y Casos Clínicos se publicarán una vez al año en un espacio destacado de uno de los números regulares de la revista.

En nombre de la Directiva de la Sociedad, como también del Comité Editorial de la Revista, debemos agradecer la generosidad, creatividad, gentileza y comprensión que han demostrado tanto los especialistas que se incorporan, se mantienen o dejan de pertenecer al nuevo Comité Editorial. 
Desde su nacimiento la revista ha debido introducir numerosas modificaciones en su estructura y funcionamiento ${ }^{9}$, para adecuarse a los requerimientos del mundo globalizado y altamente competitivo de la publicación de artículos originales en revistas biomédicas indizadas. Por lo tanto, consideramos que esta renovación parcial del Comité Editorial es sólo un paso más que debemos dar en el desarrollo de la revista.

La Revista ha jugado un papel relevante en la difusión del conocimiento científico de la especialidad durante estos años. Sin embargo, esta difusión habría sido más destacada aún, si los socios hubiesen demostrado un mayor interés en publicar en la revista. Por otra parte, sólo 138 socios de un total de 355 con e-mail, respondieron la última encuesta de la Sociedad. Es muy deseable que el entusiasmo por contestar este tipo de consultas, aumente progresivamente en las próximas encuestas. Nuestro gran desafío para el futuro inmediato es lograr que las comisiones de la Sociedad trabajen organizadamente, y que las acciones encomendadas a cada uno de los miembros del nuevo comité editorial aumenten en forma significativa la cantidad y calidad de los manuscritos enviados a la Revista.

Al considerar la historia de las revistas publicadas en los 76 años de existencia de nuestra Sociedad, se constata que su capacidad de mantener vigente al menos una revista de la especialidad a lo largo de esta dilatada trayectoria, se ha basado sistemáticamente en el esfuerzo y perseverancia de un pequeño número de sus socios, siempre dispuestos a llevar a cabo la tarea, adaptándose a condiciones que han cambiado (y lo seguirán haciendo) según la época y sus circunstancias ${ }^{10}$. La detección de esta tradicionalmente escasa participación de los miembros de la Sociedad en la edición de sus revistas, nos ha llevado a plantear que deberemos acrecentar y profesionalizar la base de miembros dedicados a labores editoriales y entusiasmar a nuevos especialistas, quienes contando con capacidades y atributos para emprender estas tareas, estén dispuestos a reemplazar a quienes hasta ahora hemos desempeñado funciones directivas en el comité editorial ${ }^{9}$. Se hace entonces imprescindible planificar un continuo proceso de renovación, profesionalización o capacitación y ampliación del núcleo de especialistas que participan en el proceso editorial, si pretendemos que la revista se mantenga, se desarrolle y logre enfrentar exitosamente los nuevos desafios que ya se asoman en el horizonte. Uno de ellos está "ad portas", es el "sistema de edición en línea" ${ }^{11}$, el cual ya está siendo usado por algunos de nosotros, cuando en calidad de autores intentamos publicar en las revistas de "corriente principal". Es evidente que más temprano que tarde deberemos implementar en nuestra revista un sistema de edición electrónica en línea, y tendremos nuevamente que adaptarnos a todas las modificaciones en el proceso editorial que demandará esta innovación.

Mientras tanto, continuaremos esforzándonos por lograr que las publicaciones en la Revista Chilena de Enfermedades Respiratorias sean un producto cabal y bien logrado de la creatividad y originalidad de sus autores y transmitan a nuestros lectores la contribución de nuestra Sociedad a los avances de la especialidad.

Dr. Manuel Oyarzún G. Editor, Revista Chilena de Enfermedades Respiratorias

\section{Bibliografías}

1.- OYARZÚN M. Ingreso de las revistas biomédicas nacionales a los índices bibliográficos internacionales: desafíos y oportunidades. Rev Chil Enf Respir 2003; 19: $5-7$.

2.- TESTA J. Sistema de evaluación para ingresar a ISI. http:॥.www.scielo.cl/news/taller_scielo_2006. Consultado 21.03.06.

3.- BORZONE G, HERRERA C. Cuenta del directorio de la Sociedad Chilena de Enfermedades Respiratorias 2002. Rev Chil Enf Respir 2002; 18: 319-24.

4.- BARROS M, LEZANA V. Cuenta del directorio de la Sociedad Chilena de Enfermedades Respiratorias 2005. Rev Chil Enf Respir 2005; 21: 302-7.

5.- VALDIVIA G. Oportunidades y estrategias de investigación colaborativa en el área de las enfermedades respiratorias. Rev Chil Enf Respir 2004; 20: 69-70.
6.- SALDÍAS F e integrantes de la Comisión ad hoc, Sociedad Chilena de Enfermedades Respiratorias: Consenso Nacional 2005: Manejo de la neumonía del adulto adquirida en la comunidad. Rev Chil Enf Respir 2005; 21-67.

7.- CORRALES R. Presente y futuro de la Sociedad Chilena de Enfermedades Respiratorias. Rev Chil Enf Respir 2006; 22: 5-6.

8.- PERTUZÉ J. Criterios para publicar casos clínicos. Rev Chil Enf Respir 2006; 22: 105-7.

9.- OYARZÚN M. Décimo aniversario de la Revista Chilena de Enfermedades Respiratorias. Rev Chil Enf Respir 1995; 11: 5-6.

10.- OYARZÚN M. Evolución de las revistas sobre enfermedades respiratorias y tuberculosis en Chile. Rev Chil Enf Respir 1998; 12: 103-18.

11.- TAKENAKA R. Edición en línea. (pdf): http:॥.www. scielo.cl/news/taller_scielo_2006. Consultado 21.03.06. 